\title{
Comparison of Adverse Events With Vancomycin Diluted in Normal Saline vs Dextrose 5\%
}

\author{
Robert C. Ross, PharmD; Bridgette A. Kelly, PharmD; Rachel M. Smith, PharmD; \\ and Andrew J. Franck, PharmD, BCNSP, BCCCP
}

\begin{abstract}
Background: IV vancomycin is widely used for infections caused by Gram-positive bacteria; however, nephrotoxicity is commonly associated with its use. Clinical trials have shown an increased incidence of acute kidney injury (AKI) using normal saline (NS) for fluid resuscitation. This study evaluated differences in $\mathrm{AKI}$ and other patient outcomes associated with vancomycin diluted in NS compared with dextrose $5 \%$ in water (D5W).
\end{abstract}

Methods: This retrospective cohort study conducted at a single US Department of Veterans Affairs hospital included veterans who received vancomycin for at least 48 hours between July 1, 2015 and June 30, 2020. This study compared adverse events (AEs) of patients receiving vancomycin diluted in either NS or D5W. The primary outcome was incidence of AKI. Secondary outcomes included incidence of hyperglycemia, hy- perchloremia, hypernatremia, metabolic acidosis, in-hospital mortality, and 30-day posthospitalization mortality.

Results: The study included 123 patients in each group $(\mathrm{N}=246)$. The percentage of AKI was $22.8 \%$ in the D5W group compared with $14.6 \%$ the NS group $(P=.14)$. There were no significant differences in the rates of hyperglycemia, hyperchloremia, hypernatremia, or metabolic acidosis between the 2 groups. In-hospital mortality and 30-day posthospitalization mortality were similar between the groups.

Conclusions: This study comparing the AEs of IV vancomycin diluted in NS and D5W found no significant differences in AKI or other patient outcomes. These study results do not suggest the crystalloid used to dilute IV vancomycin is associated with differences in nephrotoxicity or other relevant AEs.
Author affiliations can be found at the end of the article. Correspondence: Robert Ross (robert.ross1@va.gov)

Fed Pract. 2021;38(10). Published online October 12. doi:10.12788/fp.0193
V ancomycin is a widely used IV antibiotic due to its broad-spectrum of activity, bactericidal nature, and low rates of resistance; however, adverse effects (AEs), including nephrotoxicity, are commonly associated with its use. ${ }^{1}$ The vancomycin therapeutic monitoring guidelines recognize the incidence of nephrotoxicity and suggest strategies for reducing the risk, including area under the curve/mean inhibitory concentration (AUC/MIC) monitoring rather than trough-only monitoring. Vancomycin-associated acute kidney injury (AKI) has been defined as an increase in serum creatinine (SCr) over a 48-hour period of $\geq 0.3 \mathrm{mg} / \mathrm{dL}$ or a percentage increase of $\geq$ $50 \%$, which is consistent with the Acute Kidney Injury Network (AKIN) guidelines. ${ }^{2,3}$ Vancomycin-associated AKI is a common $\mathrm{AE}$, with its incidence reported in previous studies ranging from 10 to $20 \%$.,

The most common crystalloid fluid administered to patients in the United States is $0.9 \%$ sodium chloride $(\mathrm{NaCl})$, also known as normal saline (NS), and recent trials have explored its potential to cause AEs. ${ }^{6-8}$ Balanced crystalloid solutions, such as Plasma-Lyte and lactated Ringer's solution (LR), contain buffering agents and lower concentrations of sodium and chloride compared with that of NS. Trials in the intensive care unit (ICU) and emergency department, such as the SMART-MED, SMART-SURG, and SALT-
ED have reported a significantly lower rate of AKI when using balanced crystalloids compared with NS due to the concentration of sodium and chloride in NS being supraphysiologic to normal serum concentrations. ${ }^{6,7} \mathrm{Al}-$ ternatively, the SPLIT trial evaluated the use of NS compared with Plasma-Lyte for ICU fluid therapy and did not find a statistically significant difference in AKI. ${ }^{8}$ Furthermore, some studies have reported increased risk for hyperchloremia when using NS compared with dextrose $5 \%$ in water (D5W) or balanced crystalloids, which can result in metabolic acidosis. ${ }^{6,7,9,10}$ These studies have shown how the choice of fluid can have a large effect on the incidence of AEs; bringing into question whether these effects could be additive when combined with the nephrotoxicity associated with vancomycin. ${ }^{6-9}$

Vancomycin is physically and chemically stable if diluted in D5W, NS, 5\% dextrose in NS, LR, or 5\% dextrose in LR. ${ }^{1}$ It is not known whether the selection of diluent has an effect on nephrotoxicity or other AEs of vancomycin therapy. Furthermore, clinicians may be unaware or unable to specify which diluent to use. There are currently no practice guidelines that favor one diluent over another for vancomycin; however, trials showing higher rates of AKI and hyperchloremia using NS for fluid resuscitation may indicate an increased potential for vancomycin-associated AKI when using NS as a diluent. ${ }^{6,79}$ This study was 
TABLE 1 Baseline Characteristics of Study Patients by Solution

\begin{tabular}{|c|c|c|}
\hline Characteristics & Vancomycin in D5W $(n=123)$ & Vancomycin in NS $(n=123)$ \\
\hline Age, median (IQR), y & $73.0(68.0,80.5)$ & $66.0(60.0,74.0)$ \\
\hline Sex, male, No. (\%) & $116(94.3)$ & $123(100)$ \\
\hline \multicolumn{3}{|l|}{ Race/ethnicity, No. (\%) } \\
\hline White & $103(83.7)$ & $91(74.0)$ \\
\hline African American & $10(8.1)$ & $16(13.0)$ \\
\hline Hispanic & $4(3.3)$ & $3(2.4)$ \\
\hline Native Hawaiian & $0(0.0)$ & $6(4.9)$ \\
\hline Unknown/declined to answer & $6(4.9)$ & $7(5.7)$ \\
\hline \multicolumn{3}{|l|}{ Patient location, No. (\%) } \\
\hline Intensive care unit & $50(40.7)$ & $46(37.4)$ \\
\hline Step-down unit & $28(22.8)$ & $18(14.6)$ \\
\hline General medicine floor & $45(36.6)$ & $59(48.0)$ \\
\hline \multicolumn{3}{|l|}{ Metabolic levels, median (IQR), mg/dL } \\
\hline Serum creatinine & $1.1(0.9,1.5)$ & $1.1(0.8,1.5)$ \\
\hline Serum glucose & $135.0(106.0,164.0)$ & $133.0(108.0,191.5)$ \\
\hline Serum sodium & $138.0(136.0,140.0)$ & $137.0(135.0,140.0)$ \\
\hline Serum chloride & $101.0(97.0,104.0)$ & $101.0(98.0,103.0)$ \\
\hline Serum bicarbonate & $23.0(21.0,25.0)$ & $23.0(20.5,25.0)$ \\
\hline Concomitant piperacillin/tazobactam, No. (\%) & $34(27.6)$ & $50(40.7)$ \\
\hline Concomitant cefepime, No. (\%) & $56(45.5)$ & $50(40.7)$ \\
\hline Concomitant aminoglycosides, No. (\%) & $0(0.0)$ & $0(0.0)$ \\
\hline Highest vancomycin trough, median (IQR), mcg/mL & $19.0(15.5,21.7)$ & $18.0(15.0,22.2)$ \\
\hline Cumulative vancomycin dose, median (IQR), mg & $5250.0(3000.0,7500.0)$ & $7000.0(3750.0,11,250.0)$ \\
\hline Average vancomycin daily dose, median (IQR), mg & $1142.9(854.2,1500.0)$ & $1708.3(1250.0,2100.0)$ \\
\hline Duration of vancomycin therapy, median (IQR), $d$ & $4.0(3.0,7.0)$ & $4.0(3.0,7.0)$ \\
\hline
\end{tabular}

Abbreviations: D5W, 5\% dextrose in water; IQR, interquartile range; NS, normal saline.

performed to evaluate whether the type of crystalloid used (D5W vs NS) can influence adverse outcomes for patients. While many factors may contribute to these AEs, the potential to reduce the risk of negative adverse outcomes for hospitalized patients is a significant area of exploration.

The primary outcome of this study was the incidence of AKI, defined using AKIN guidelines where the increase in $\mathrm{SCr}$ occurred at least 24 hours after starting vancomycin and within 36 hours of receiving the last vancomycin dose. ${ }^{3}$ AKI was staged using the AKIN guidelines (stage 1: increase in $\mathrm{SCr}$ of $\geq 0.3 \mathrm{mg} / \mathrm{dL}$ or by 50 to $99 \%$; stage 2: increase in SCr by 100 to $199 \%$; stage 3: increase in SCr by $>200 \%$ ) based on changes in SCr from baseline during vancomycin therapy or within 36 hours of stopping vancomycin therapy. ${ }^{3}$ Secondary outcomes included the incidence of hyperglycemia, hyperchloremia, metabolic acidosis, hypernatremia, mortality in hospital, and mortality within 30 days from hospital discharge.

\section{METHODS}

This single-center, retrospective study of veterans who received IV vancomycin within the North Florida/South Georgia Veterans Health System (NF/SGVHS) in Gainesville, Florida, from July 1, 2015 to June 30, 2020, compared veterans who received vancomycin diluted in NS with those who received vancomycin diluted in D5W to assess for differences in AEs, including AKI, metabolic acidosis (serum bicarbonate level $<23 \mathrm{mmol} / \mathrm{L}$ ), hyperchloremia (serum chloride levels $>108 \mathrm{mmol} / \mathrm{L}$ ), hypernatremia (serum sodium $>145 \mathrm{mmol} / \mathrm{L}$ ), and hyperglycemia (blood glucose $>180 \mathrm{mg} / \mathrm{dL}$ ). The endpoint values were defined using the reference ranges determined by the local laboratory. At NF/SGVHS, vancomycin is diluted in D5W or NS based primarily on factors such as product availability and cost. 
TABLE 2 Adverse Effects by Solution

\begin{tabular}{lccc} 
Adverse Effects & $\begin{array}{c}\text { Vancomycin in D5W } \\
\text { No. (\%) }(\mathbf{n}=\mathbf{1 2 3})\end{array}$ & $\begin{array}{c}\text { Vancomycin in NS } \\
\text { No. (\%) }(\mathbf{n}=\mathbf{1 2 3})\end{array}$ & $\begin{array}{c}\boldsymbol{P} \\
\text { value }\end{array}$ \\
\hline Acute kidney injury & $28(22.8)$ & $18(14.6)$ & .14 \\
\hline Hyperglycemia & $40(32.5)$ & $49(39.8)$ & .29 \\
\hline Hypernatremia & $19(15.4)$ & $13(10.6)$ & .34 \\
\hline Hyperchloremia & $28(22.8)$ & $22(17.9)$ & .43 \\
\hline Metabolic acidosis & $59(48.0)$ & $61(49.6)$ & .90 \\
\hline Hospital mortality & $6(4.9)$ & $7(5.7)$ & .78 \\
\hline 30-d posthospitalization mortality & $10(8.5)$ & $5(4.5)$ & .30
\end{tabular}

Abbreviations: D5W: $5 \%$ dextrose in water; NS: normal saline.

\section{Study Criteria}

Veterans were included if they received IV vancomycin between July 1, 2015 and June 30, 2020. The cohorts were grouped into those receiving vancomycin doses diluted in NS and those receiving vancomycin doses diluted in D5W. Veterans were excluded if they received $<80 \%$ of vancomycin doses diluted in their respective fluid, if they were on vancomycin for $<48$ hours, or if they did not have laboratory results collected both before and after vancomycin therapy to assess a change. There were more patients receiving vancomycin in $\mathrm{D} 5 \mathrm{~W}$, so a random sample was selected to have an equal size comparison group with those receiving NS. A sample size calculation was performed with an anticipated AKI incidence of $14 \% .{ }^{5}$ To detect a $10 \%$ difference in the primary outcome with an $\alpha$ of 0.05 and $75 \%$ power, 226 patients (113 in each cohort) were needed for inclusion.

Data were collected using the Data Access Request Tracker tool through the US Department of Veterans Affairs (VA) Informatics and Computing Infrastructure. Data collected included demographics, laboratory data at baseline and during vancomycin therapy, characteristics of antibiotic therapy, and mortality data. Of note, all laboratory values assessed in this study were obtained while the veteran was receiving vancomycin or within 36 hours of receiving the last vancomycin dose to appropriately assess any changes.

Statistical analysis of categorical data were analyzed using a $\chi^{2}$ test on the GraphPad online program. This study received institutional review board approval from the
University of Florida and was conducted in accordance with protections for human subjects.

\section{RESULTS}

A total of 792 veterans received IV vancomycin NF/SGVHS in the defined study period. Of these, 381 veterans were excluded, including having $<80 \%$ of doses in a single solution (213 veterans), receiving IV vancomycin for $<48$ hours (149 veterans), and not having necessary laboratory data available to assess a change in kidney function (19 veterans). An additional 165 veterans were randomly excluded from the D5W cohort in order to have an equal comparison group to the NS cohort; therefore, a total of 246 veterans were included in the final assessment (123 veterans in each cohort). The median patient age was 73 years (IQR, 68.0, 80.5 ) in the D5W group and 66 years (IQR, $60.0,74.0$ ) in the NS group; $83.7 \%$ of veterans in the D5W group and $74 \%$ veterans in the NS group were white; $94.3 \%$ of the D5W group and $100 \%$ of the NS group were male (Table 1).

\section{Adverse Effects by Solution}

The percentage of AKI in the D5W group was $22.8 \%$ compared with $14.6 \%$ in the NS group $(P=.14)$, and all cases were classified as stage 1 AKI. Baseline cases of hyperglycemia, hypernatremia, hyperchloremia, or metabolic acidosis were not included in the reported rates of each in order to determine a change during vancomycin therapy (Table 2 ).

The percentage of patients with hyperglycemia in the D5W group was $32.5 \%$ compared with $39.8 \%$ in the NS group $(P=.29)$. The percentage of patients with hypernatremia in the D5W group was $15.4 \%$ compared with $10.6 \%$ in the NS group $(P=.34)$. The percentage of patients with hyperchloremia in the D5W group was $22.8 \%$ compared with $17.9 \%$ in the NS group $(P=.43)$. The percentage of patients with metabolic acidosis in the D5W group was $48.0 \%$ compared with $49.6 \%$ in the NS group $(P=.90)$.

There were no significant differences in either in-hospital or posthospital mortality between the D5W and NS groups (in-hospital: $4.9 \%$ vs $5.7 \%$, respectively; $P=.78 ; 30$-day posthospitalization: $8.5 \%$ vs $4.5 \%$, respectively; $P=.30$ ). 


\section{DISCUSSION}

This retrospective cohort study comparing the AEs of vancomycin diluted in NS and vancomycin diluted with D5W showed no statistically significant differences in the incidence of AKI or any metabolic AEs. Although these results did not show an association between the incidence of AEs and the dilution fluid for vancomycin, other factors may contribute to the overall incidence of AEs. Factors such as cumulative vancomycin dose, duration of therapy, and presence of concomitant nephrotoxins have been known to increase the incidence of AKI and may have a greater impact on this incidence than the fluid used in administering the vancomycin.

These results specifically the incidence of AKI were not consistent with previous trials evaluating the AEs of NS. Based on previous trials, we expected the vancomycin in the NS cohort to have a significantly higher incidence of hypernatremia, hyperchloremia, and AKI. Our results may indicate that the volume of crystalloid received played a greater role on the incidence of AEs. Our study assessed the effect of a diluent for one IV medication that may have been only a few hundred milliliters of fluid per day. The total volume of IV fluid received from vancomycin was not assessed; thus, it is not known how the volume of fluid may have impacted the results.

One consideration with this study is the method used for monitoring vancomycin levels. Most of the patients included in this study were admitted prior to the release of the updated vancomycin guidelines, which advocated for the transition from traditional trough-only monitoring to AUC/MIC. In September 2019, NF/SGVHS ICUs made the transition to this new method of monitoring with a hospital-wide transition following the study end date. The D5W group had a slightly higher percentage of patients admitted to the ICU, thus were more likely to be monitored using AUC/MIC during this period. Literature has shown the AUC/MIC method of monitoring can result in a decreased daily dose, decreased trough levels, and decreased incidence of nephrotoxicity. ${ }^{11-14}$ Although the method for monitoring vancomycin has the potential to affect the incidence of AKI, the majority of patients were monitored using the traditional trough- only method with similar trough levels reported in both groups.

\section{Limitations}

This study is limited by its retrospective nature, the potential introduction of biases, and the inability to control for confounders that may have influenced the incidence of AEs. Potential confounders present in this study included the use of concomitant nephrotoxic medications, vancomycin dose, and underlying conditions, as these could have impacted the overall incidence of AEs.

The combination of piperacillin/tazobactam plus vancomycin has commonly been associated with an increased risk of nephrotoxicity. Previous studies have identified this nephrotoxic combination to have a significantly increased risk of AKI compared with vancomycin alone or when used in combination with alternative antibiotics such as cefepime or meropenem..$^{15,16}$ In our study, there was a higher percentage of patients in the NS group with concomitant piperacillin/tazobactam, so this difference between the groups may have influenced the incidence of AKI. Nephrotoxic medications other than antibiotics were not assessed in this study; however, these also could have impacted our results significantly. While the vancomycin duration of therapy and highest trough levels were similar between groups, the NS group had a larger average daily dose and overall cumulative dose. Studies have identified the risk of nephrotoxicity increases with a vancomycin daily dose of $4 \mathrm{~g}$, troughs $>15 \mathrm{mg} / \mathrm{mL}$, and a duration of therapy $>7$ days. ${ }^{15,16}$ In our study, the daily doses in both groups were $<4 \mathrm{~g}$, so it is likely the average daily vancomycin dose had little impact on the incidence of AKI.

Another potential confounder identified was assessment of underlying conditions in the patients. Due to the limitations associated with the data extraction method, we could not assess for underlying conditions that may have impacted the results. Notably, the potential nephrotoxicity of NS has mostly been shown in critically ill patients. Therefore, the mixed acutely ill patient sample in this study may have been less likely to develop AKI from NS compared with an exclusively critically ill patient sample.

Selection bias and information bias are common with observational studies. In our 
study, selection bias may have been present since prospective randomization of patient samples was not possible. Since all data were extracted from the medical health record, information bias may have been present with the potential to impact the results. Due to the single-center nature of this study with a predominantly older, white male veteran patient sample, generalizability to other patient populations may be limited. We would expect the results of this study to be similar among other patient populations of a similar age and demographic; however, the external validity of this study may be weak among other populations. Although this study included enough patients based on sample size estimate, a larger sample size could have allowed for detection of smaller differences between groups and decreased the chance for type II error.

\section{CONCLUSIONS}

Overall, the results of this study do not suggest that the crystalloid used to dilute IV vancomycin is associated with differences in nephrotoxicity or other relevant AEs. Future studies evaluating the potential for AEs from medication diluent are warranted and would benefit from a prospective, randomized design. Further studies are both necessary and crucial for enhancing the quality of care to minimize the rates of AEs of commonly used medications.

\section{Author affiliations}

Robert Ross is a Clinical Pharmacy Fellow, Bridgette Kelly is a Pharmacy Resident, Rachel Smith is a Pharmacy Resident, and Andrew Franck is a Clinical Pharmacy Specialist at the North Florida/South Georgia Veterans Health System in Gainesville, Florida.

\section{Acknowledgment}

This material is the result of work supported with resources and the use of facilities at the North Florida/South Georgia Veterans Health System in Gainesville, Florida.

\section{Author disclosures}

The authors report no actual or potential conflicts of interest with regard to this article.

\section{Disclaimer}

The opinions expressed herein are those of the authors and do not necessarily reflect those of Federal Practitioner, Frontline Medical Communications Inc., the US Government, or any of its agencies. This article may discuss unlabeled or investigational use of certain drugs. Please review the complete prescribing information for specific drugs or drug combinations-including indications, contraindications, warnings, and adverse effectsbefore administering pharmacologic therapy to patients.

\section{References}

1. Vancomycin hydrochloride intravenous injection, pharmacy bulk package. Package insert. Schaumburg, IL: APP Pharmaceuticals, LLC; 2011.
2. Rybak MJ, Le J, Lodise TP, et al. Therapeutic monitoring of vancomycin for serious methicillin-resistant Staphylococcus aureus infections: a revised consensus guideline and review by the American Society of Health-System Pharmacists, the Infectious Diseases Society of America, the Pediatric Infectious Diseases Society, and the Society of Infectious Diseases Pharmacists. Am J Health-System Pharm. 2020;77(11):835-864. doi:10.1093/ajhp/zxaa036

3. Mehta RL, Kellum JA, Shah SV, et al; Acute Kidney Injury Network. Acute Kidney Injury Network: report of an initiative to improve outcomes in acute kidney injury. Crit Care. 2007;11(2):R31. doi:10.1186/cc5713

4. Elaysi S, Khalili H, Dashti-Khavidaki S, Mohammadpour A. Vancomycin-induced nephrotoxicity: mechanism, incidence, risk factors and special populations-a literature review. Eur J Clin Pharmacol. 2012;68(9):1243-1255. doi:10.1007/s00228-012-1259-9

5. Gyamlani G, Potukuchi PK, Thomas F, et al. Vancomycin-associated acute kidney injury in a large veteran population. Am J Nephrol. 2019;49(2):133-142. doi:10.1159/000496484

6. Semler MW, Self WH, Wanderer JB, et al; SMART Investigators and the Pragmatic Critical Care Research Group. Balanced crystalloids versus saline in critically ill adults. N Engl Med. 2018;378(9):829-839. doi:10.1056/NEJMoa1711584

7. Self WH, Semler MW, Wanderer JP, et al; SMART Investigators and the Pragmatic Critical Care Research Group. Balanced crystalloids versus saline in noncritically ill adults. N Engl J Med. 2018;378(20):819-828. doi:10.1056/NEJMc1804294

8. Young P, Bailey M, Beasley R, et al; SPLIT Investigators; ANZICS CTG. Effect of a buffered crystalloid solution vs saline on acute kidney injury among patients in the intensive care unit: the SPLIT Randomized Clinical Trial. JAMA. 2015;314(16):1701-1710. doi:10.1001/jama.2015.12334

9. Magee CA, Bastin ML, Bastin T, et al. Insidious harm of medication diluents as a contributor to cumulative volume and hyperchloremia: a prospective, open-label, sequential period pilot study. Crit Care Med. 2018;46(8):1217-1223. doi:10.1097/CCM.0000000000003191

10. Adeva-Andany $M M$, Fernández-Fernández $C$, Mouriño-Bayolo D, Castro-Quintela E, DomínguezMontero A. Sodium bicarbonate therapy in patients with metabolic acidosis. ScientificWorldJournal. 2014;2014:627673. doi:10.1155/2014/627673

11. Mcgrady KA, Benton M, Tart S, Bowers R. Evaluation of traditional vancomycin dosing versus utilizing an electronic AUC/MIC dosing program. Pharm Pract (Granada). 2020;18(3):2024. doi:10.18549/PharmPract.2020.3.2024

12. Clark L, Skrupky LP, Servais R, Brummitt CF, Dilworth TJ. Examining the relationship between vancomycin area under the concentration time curve and serum trough levels in adults with presumed or documented staphylococcal infections. Ther Drug Monit. 2019;41(4):483-488. doi:10.1097/FTD.0000000000000622

13. Neely MN, Kato L, Youn G, et al. Prospective trial on the use of trough concentration versus area under the curve to determine therapeutic vancomycin dosing. Antimicrob Agents Chemother. 2018;62(2):e02042-17. doi:10.1128/AAC.02042-17

14. Aljefri DM, Avedissian SN, Youn G, et al. Vancomycin area under the curve and acute kidney injury: a meta-analysis. Clin Infect Dis. 2019;69(11):1881-1887. doi:10.1128/AAC.02042-17

15. Molina KC, Barletta JF, Hall ST, Yazdani C, Huang V. The risk of acute kidney injury in critically ill patients receiving concomitant vancomycin with piperacillin-tazobactam or cefepime. J Intensive Care Med. 2019;35(12):1434-1438. doi:10.1177/0885066619828290

16. Burgess LD, Drew RH. Comparison of the incidence of vancomycin-induced nephrotoxicity in hospitalized patients with and without concomitant piperacillin-tazobactam. Pharmacotherapy. 2014; 34(7):670-676. doi:10.1002/phar.1442 